\title{
A Lateral Modes Model for BAW Resonators
}

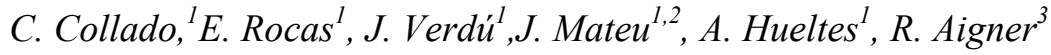 \\ ${ }^{1}$ Universitat Politècnica de Catalunya (UPC), Barcelona, Spain \\ ${ }^{2}$ Centre Tecnologic de Telecomunicacions de Catalunya (CTTC), Castelldefels, Spain \\ ${ }^{3}$ TriQuint Semiconductor, Apopka, FL, USA
}

\begin{abstract}
An equivalent circuit model to predict lateral modes occurring in real BAW resonators is proposed. The circuit model has been validated with FEM simulations of FBAR resonators and also with measurements of SMR resonators. The model allows to predict the electrical response of square-shaped and rectangular-shaped resonators without limitation on dimensions.
\end{abstract}

Keywords-Bulk Acoustic Wave, resonator, lateral modes, circuit model.

\section{INTRODUCTION}

Finite dimensions of BAW resonators originate Lamb waves to laterally propagate, creating visible resonant modes in the in-band response of BAW filters, thus degrading its ideal expected performance. The 3D nature of these lateral modes makes inherently complex to predict their resonance frequencies, and most of previous circuit modeling efforts found in literature are based on phenomenological lumped models fitted to pre-computed FEM solutions. These previous modeling approaches lead to circuit implementations with many parallel RLC branches, one for each mode, shunt connected to the BVD model [1], thus becoming dimensionspecific models not able for prediction. Reference [2] uses a transmission line model for a given lateral resonance which is coupled to the 1D Mason model that simulates the fundamental resonance "piston mode".

The method presented below uses the analogy between acoustic plate waves and electromagnetic waveguides to account for the lateral modes occurring in BAW resonators. The electromagnetic waveguides are analyzed through the transverse resonance method, which allows to treat independently both in-plane spatial directions. Propagating modes are defined in the transverse direction, which sets their cut-off frequency according to the lateral and thickness dimensions, as well as phase velocity and mode order. These modes are then allowed to propagate in the other in-plane direction by implementing a transmission line circuit for each propagating mode, so that each transverse mode will show its harmonic resonances in the other direction. These transmission lines make use of the dispersive phase constant along with the cut-off frequency obtained from the transverse analysis to model the Lamb waves.

The novelty of this method is the used of the distributed nature of a transmission line to account for several resonances (lateral modes), which indeed reduces the number of parameters of the model.

\section{LAMB WAVES}

In order to model the propagation of Lamb-waves as dispersive transmission lines we need to state the analogy between voltage-current and velocity-stress.

Lamb waves are formed at the interfaces of a piezoelectric sheet due to the coupling between the longitudinal and transversal vertical propagating waves. Mathematical derivation of the displacement and stress distribution can be derived for nonpiezoelectric isotropic plates as shown in [3],[4]. That should be enough to provide some clues about how we can formulate an equivalent transmission line model that should be able to simulate the propagation of Lamb waves even in more complex structures, such as FBAR resonators or even SMR resonators.

\section{A. Displacement and Stress}

The displacement field distribution in an isotropic plate (thickness defined in $z$-axis and infinite in the y-direction) of a Lamb wave propagating in the $\mathrm{x}$-direction can be written as [4]:

$$
\begin{aligned}
& u_{x}=(q A \cos (q z) \mp j \beta B \cos (p z)) e^{\mp j \beta x} \\
& u_{z}=(-p B \sin (p z) \pm j \beta A \sin (q z)) e^{\mp j \beta x} .
\end{aligned}
$$

Where $p$ and $q$ are respectively defined as $p^{2}=\omega^{2} / V_{L}^{2}-\beta^{2}$ and $q^{2}=\omega^{2} / V_{T}^{2}-\beta^{2}$, being $V_{L}$ and $V_{T}$ the phase velocities of the longitudinal and transverse waves, respectively. The Lamb wave propagates with a phase constant $\beta$ that must fulfill the Rayleigh-Lamb equation

$$
\frac{\omega^{4}}{V_{T}^{4}}=4 q^{2} \beta^{2}\left(1-\frac{p \tan (p t / 2)}{q \tan (q t / 2)}\right) .
$$

at a given frequency $\omega$. Additionally the boundary conditions in the free space surfaces at $z= \pm t / 2$ impose that

$$
B=-2 \frac{j \beta A q}{\beta^{2}-q^{2}} \frac{\cos (q t / 2)}{\cos (p t / 2)} .
$$

Note that the expressions above correspond to the specific symmetric Lamb-wave modes in which we are interested. The solutions of (1)-(3) will give rise to the displacement of all the symmetric $S_{n}$ Lamb-wave modes for $\mathrm{n}=0,1,2 \ldots$, following the nomenclature in [4]

For example, if we assume $\mathrm{V}_{\mathrm{L}} / 2>\mathrm{V}_{\mathrm{T}}$, as is the case of $\mathrm{ZnO}$ resonators, the solutions of (2) gives the dispersive curves of Fig. 1 [3] for a thickness $t=1.52 \mu \mathrm{m}$.

For a $\mathrm{ZnO}$ FBAR resonator (Type I resonator) with lateral modes above the series resonance, we are interested in the S1 mode. 


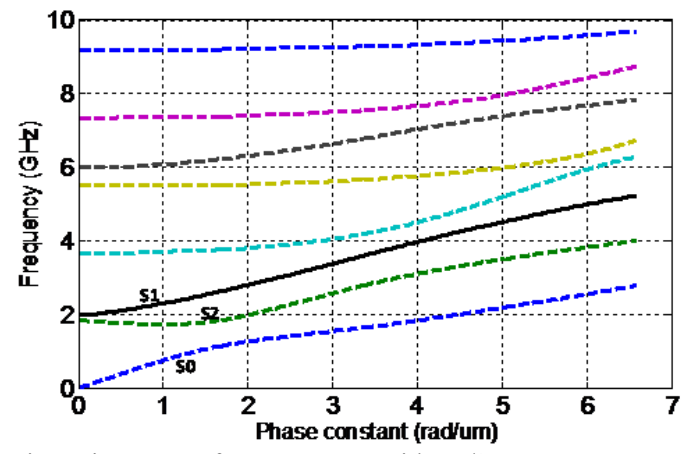

Fig. 1. Dispersive curves for a resonator with $V_{L} / 2>V_{T}$.

At very low wavenumbers $(\beta \sim 0)$ and for the $\mathrm{S} 1$ odd mode, A $\sim 0$ and (1) simplifies as:

$$
u_{x} \approx 0 ; u_{z}=-p B \sin (p z) e^{\mp j \beta x}
$$

and then the stress $T_{x z}$ will have the same pattern distribution than $u_{z}$ in the z-direction given by

$$
T_{y z}=c_{44}( \pm 2 j \beta p B) \sin (p z) e^{\mp j \beta x},
$$

being $c_{44}$ the shear modulus.

In practical situations, the wave numbers of the lateral modes are large enough to consider that the displacement strictly follows the pattern outlined by (4). Figure 2 uses (1)-(4) to obtain the displacement $u_{z}$ and $u_{x}$ at the wave number $\beta=5 \pi / a$ $(a=100 \mu \mathrm{m})$. The constant $A$ is set to $10^{-6}$. As can be seen the assumption that $u_{x} \sim 0$ (in (4)) is no longer valid and the component $u_{x}$ of the displacement becomes significant having an even distribution in the $\mathrm{z}$-direction.

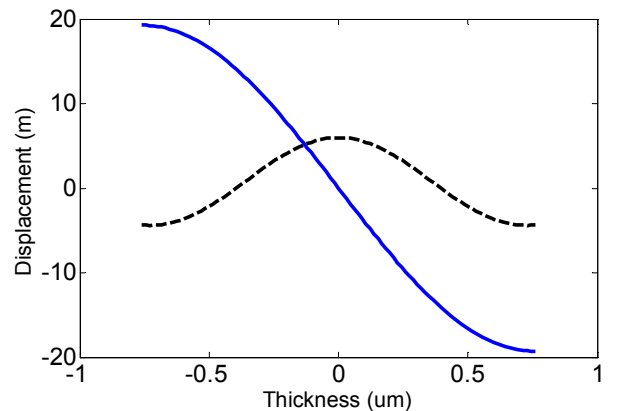

Fig. 2. Displacement $u_{z}$ (continuous) and $u_{x}$ (dotted) at $\beta=5 \pi / a$, with $a=100$ $\mu \mathrm{m}$. Constant $A$ in (1) and (3) is set to $10^{-6}$.

In spite of that, we may assume that this component will not manifest in the electrical response of a resonator due to its even symmetry, being therefore valid the phenomenological equivalent circuit outlined by (4) and (5). Note that these equations will determine the characteristic impedance of the lateral transmission lines to be used in our model

\section{LATERAL TRANSMISSION LINES}

In contrast with the isotropic infinite plate case depicted in previous section II, this section addresses the case of a piezoelectric finite problem. Although it is indeed a much more complicated configuration to analyze, a useful phenomenological equivalent circuit is proposed.

The equivalent circuit model results in a set of lateral transmission lines with its corresponding resonances coupled to the conventional Mason model. The procedure to find the resonant frequencies and coupling values are described in subsections below.

\section{A. Resonance Frequencies of the Lateral Modes}

The method proposed uses the analogy with electromagnetic rectangular waveguides to model the lateral modes of rectangular BAW resonators, with in-plane dimensions $a$ and $b$. The procedure considers that a set of modes (standing wave patterns), which are defined in the transverse direction (for example the " $a$-direction"), propagate in the other in-plane " $b$ direction" with a dispersive phase constant with its corresponding phase velocity and cut-off frequency according to the lateral and thickness $t$ dimensions.

We consider that the phase constant of the $m$ propagating mode $(m=1,3,5, .$.$) , follows the expression given by:$

$$
\beta_{m}=\frac{2 \pi}{c_{\text {lamb }}}\left(f^{k}-f_{m 01}^{k}\right)^{1 / k},
$$

where the exponent $k$ (usually close to 2 ) and the constant $c_{\text {lamb }}$ are obtained experimentally, and are the same for all the $m$ modes. The cut-off frequency $f_{m 01}$ indicates that the standing wave pattern in the transversal direction is $m$-times a half wavelength, the subscript 0 indicates the propagating direction, and the subscript 1 indicates a half wavelength in the thickness direction. Then resulting in

$$
f_{m 01}^{k}=\left(c_{\text {lamb }} \frac{m}{2 a}\right)^{k}+\left(f_{001}\right)^{k},
$$

where $f_{001}$ is the frequency of the pure thickness mode, the so called "piston mode", and $a$ is the width in which the modes are defined. This $m$-mode propagates in the $b$-direction creating lateral resonances when $\beta_{m}=n \pi / b$ for $n=1,3,5$.., with $b$ being the other in-plane dimension of the rectangular resonator. Following this procedure it may be concluded that the frequencies where the lateral resonances occur are:

$$
f_{m n 1}^{k}=\left(c_{\text {lamb }} \frac{m}{2 a}\right)^{k}+\left(c_{\text {lamb }} \frac{n}{2 b}\right)^{k}+\left(f_{001}\right)^{k} .
$$

Eigen-mode finite element 3D simulations of FBAR resonators confirm that the equation above may be used to accurately predict the lateral resonance frequencies just by fitting the parameters $k$ and $c_{\text {lamb }}$, even for high order modes. In order to obtain a phenomenological full equivalent circuit model it is also necessary to predict the coupling of the lateral modes, not only their frequencies. Details on that are given below.

\section{B. Lateral Transmission Line (LTL) Model}

A dispersive transmission line with phase constant $\beta_{m}$ (as in (6)) is used to simulate the lateral modes associated to a given $m$-mode defined in the $a$-direction. In an electrical analogy the velocity $v_{z}$ is accounted by the current and the force $A_{r} \cdot T_{y z}$ by the voltage, being $A_{r}$ the cross-section area of the resonator.

The characteristic impedance $Z_{m}$ of this transmission line is then obtained by the use of (4) and (5),

$$
Z_{m}= \pm 2 \beta_{m} \frac{c_{44}}{\omega} A_{r} \text {. }
$$


Note that for modes defined in the $b$-direction that propagates in the $a$-direction equivalent equations are used just replacing $a$ by $b$.

\section{Coupling of the LTL's}

Separation of spatial variables is used to solve for the coupling of the lateral mode, following the idea described in [5], in which the coupling in all directions is considered independently. As conventionally done, we have used the Mason model for modelling the BAW resonator without lateral effects. Then, each LTL, one for each propagating mode, is coupled by means of an electrical transformer into the acoustic domain of the Mason model, in a similar way as done in [2]. Outline of the equivalent circuit is given in Fig. 3. Doing this way, the strength of the coupling depends on the velocity of the particles for the main mode ("piston-mode"), which is equivalent to consider the coupling in the thickness direction.

The coupling in the propagating direction (for example the $b$ direction) of all resonating modes that correspond to a given propagating mode (a given LTL) is achieved automatically by the distributed nature of the LTL. To avoid the coupling of the even modes we have short-ended the LTL (see Fig. 3). That means that the short-end point of the LTL corresponds to a point with maximum displacement (maximum current), that is, the center of the resonator. Note then that the resonators are coupled by one end, being the length of the transmission line $b / 2$. The symmetry of a rectangular resonator makes unneeded to model the other half resonator.

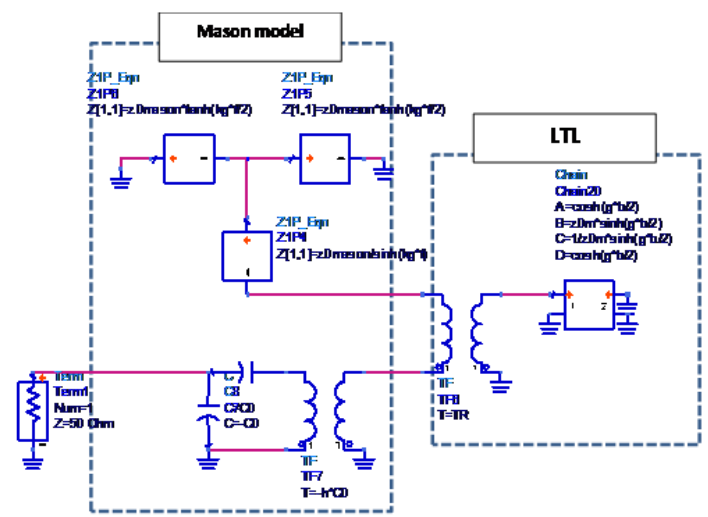

Fig. 3. Circuit model of a lateral transmission line (represented by its ABCD matrix) connected to a conventional Mason model

The coupling in the other in-plane direction (the $a$-direction in this case), is the coupling of the standing wave pattern associated to each LTL. To take into account this effect we used the formula given in [5], which assumes pure standingwave displacement patterns of the modes:

$$
k_{y}^{2}=\frac{8}{\pi^{2}} \frac{1}{(2 m+1)^{2}} ; m=0,1,2 .
$$

This coefficient scales the turn ratio $(T R)$ of the electrical transformer for each LTL depending on the order $m$ of the mode. Then the coupling of all LTLs and thus all lateral modes, will depend only of one constant, the turn ratio of all electrical transformers, which is scaled by (10). This constant $T R$ is fitted experimentally.

\section{PRACTICAL CONSIDERATION FOR RECTANGULAR RESONATORS}

\section{A. Superposition of modes}

Figure 4 shows the displacement $u_{z}$ at the surface of a membrane resonator obtained by 3D FEM simulations at the frequency that corresponds to the mode 131. As can be seen, the resulting pattern is the superposition of the mode 131 defined in the $a$-direction and the mode 131 defined in the $b$ direction, both of them are obviously at the same frequency because $a=b$ for a square resonator. That implies that for rectangular resonator, a general model must include a set of LTL for modes defined in the $a$-direction and other set for modes defined in the $b$-direction.

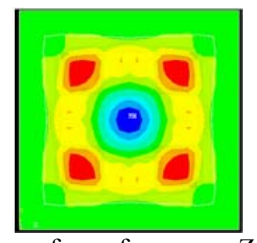

Fig. 4. Displacement at the surface of a square $\mathrm{ZnO}$ membrane resonator at the frequency of the mode 131 .

\section{B. Cut-off frequency as a function of series resonance.}

The main mode is the 111 mode and its frequency is slightly higher than the pure piston mode 001 . The frequency of the 111 mode corresponds to the measured (or simulated) series resonance and it is usually an input parameter to the model in practical situations. It is convenient then, to calculate the frequency $f_{001}$ as a function of the series resonance to avoid adjusting a new parameter in the dispersive phase of the LTL's, which can be obtained from (6)-(8). Therefore, only two parameters are required in the definition of the dispersive phase constants: the exponent $k$ and the phase velocity $c_{\text {lamb }}$.

\section{Losses}

The LTLs are considered lossy transmission lines with an attenuation constant $\alpha$, which is constant with the frequency in the range of frequencies of interest. This is a third constant that must be included in the model of LTLs.

\section{Characteristic impedance}

The characteristic impedance does not introduce any additional parameter into the model. Although the expression for the characteristic impedance is given in (9), in practical situations involving more complex structure, it might not be exactly describing the ratio between $T_{y z}$ and $v_{z}$. Its uncertainty could be absorbed by the constant $T R$ of the electrical transformers.

For cases used in this paper, the frequency dispersive behavior observed in simulations and measurements are consistent with the one in (9). As shown in the following section, such a dispersive behavior (9) should be taken into account to predict the coupling of all the lateral modes between the series resonance and the shunt resonance of a type I resonator.

\section{SIMULATIONS AND MEASUREMENTS}

\section{A. 3D FEM simualtions of FBAR resonators}

$2 \mathrm{D}$ and 3D FEM simulations of a square-shaped $\mathrm{ZnO}$ resonator with dimension $100 \mu \mathrm{m} \times 100 \mu \mathrm{m} \times 1.52 \mu \mathrm{m}$ have 
been performed to validate the circuit model above. The loss factor for elasticity matrix of the $\mathrm{ZnO}$ has been artificially lowered to $2 \cdot 10^{-4}$ to clearly emphasize the lateral modes in its electrical response. Figure 5 shows the input impedance of the 2D, 3D FEM simulation and the adjustment of the circuit model proposed. The parameters that offer a best fitting with the FEM simulations are $k=1.96, c_{\text {lamb }}=6870 \mathrm{~m} / \mathrm{s}, \alpha=1600$ $\mathrm{Np} / \mathrm{m}$ and $T R=\operatorname{sqrt}(2)$. Note that $T R$ would be 1 if we would consider two identical LTL in the model in Fig 3 to simulate the other half-resonator, instead.
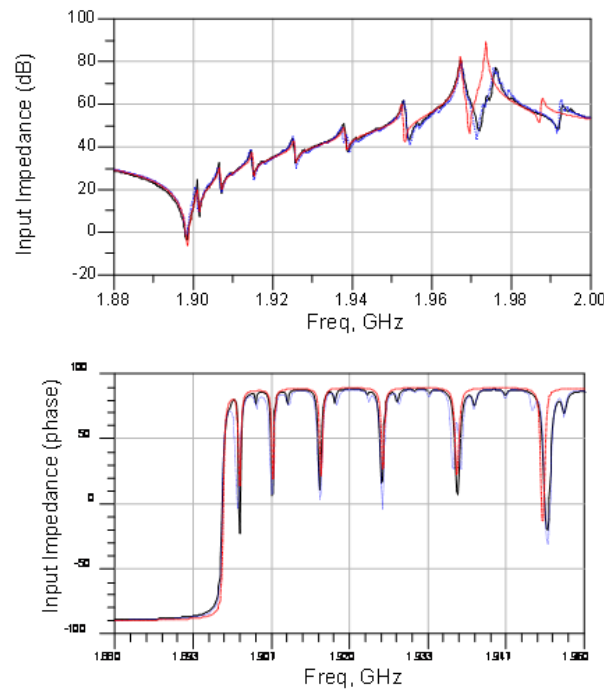

Fig. 5. Input impedance of a $\mathrm{ZnO}$ square resonator. 2D FEM simulations are plotted in red, 3D simulations in dotted blue line. The results of the circuit model are plotted in continuous black line.

For a square-shaped resonator only the LTLs that simulate modes defined in one in-plane direction would be required since $a=b$. However, in this simulation we used three LTLs for modes $1 m 1,3 m 1$ and $5 m 1$, respectively, and three additional identical LTLs for the modes defined in the other direction.

As it can be seen in Fig. 5, the circuit model (black line) agrees very well the frequency and coupling strength of the lateral modes, even for high order modes. The most coupled modes correspond to the propagating modes $1 \mathrm{~m} 1$ so in a practical situation with realistic losses, only one LTL would be required for square resonators. Note that the 2D FEM simulations (red line) cannot simulate other propagating modes than the mode $1 m 1$.

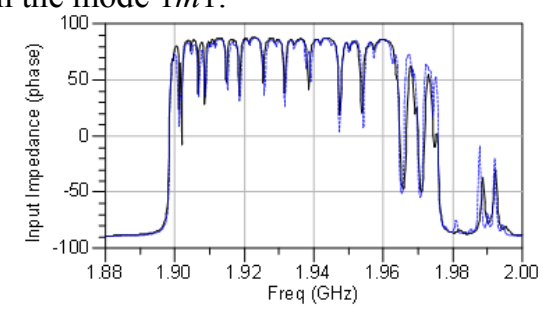

Fig. 6. Input impedance of a $\mathrm{ZnO}$ rectangular resonator. Results of 3D FEM simulation are plotted in dotted blue line. The results of the circuit model are plotted in continuous black line.

Results of a rectangular-shaped resonator with identical mechanical properties and dimensions $90 \mu \mathrm{m} \times 100 \mu \mathrm{m} \times 1.52$ $\mu \mathrm{m}$ are shown in Fig. 6 . The parameters $k, c_{\text {lamb }}, \alpha$ and $T R$ are the same than previously used for the square-shaped resonator.
In contrast with the square-shaped resonator the deepest modes now clearly split into two modes and they are less coupled than previously. Again, the model adjusts very well the FEM simulations.

Simulations with other rectangular-shaped resonators with different aspect ratio do also agree with the same adjusted parameters, validating therefore the usefulness of the circuit model proposed.

\section{B. SMR resonator measurements}

Finally the same equivalent circuit has been used to predict the electrical performance of a real SMR resonator, by comparing with real measurements. Figure 7 shows a very good agreement between simulations and measurements. In this case the parameters adjusting the measurements are $k=2.05$, $c_{\text {lamb }}=5800 \mathrm{~m} / \mathrm{s}, \alpha=7500 \mathrm{~Np} / \mathrm{m}$ and $T R=\operatorname{sqrt}(2)$. The most coupled modes are again those corresponding to the LTL $1 \mathrm{~m} 1$ and higher order modes are barely visible in the measurements and the simulations. Therefore just one LTL would be required since this resonator is square-shaped.
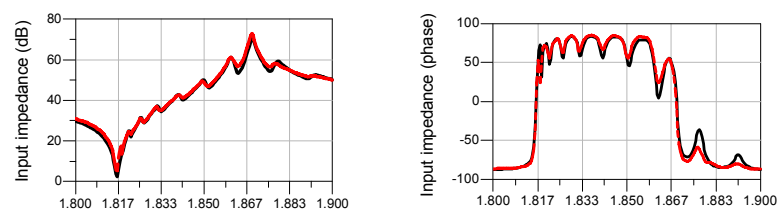

Freq $(\mathrm{GHz})$

Freq $(\mathrm{GHz})$

Fig. 7. Measured (red dotted line) input impedance of a SMR square resonator and circuit model (continuous black).

\section{DISCUSSION}

The proposed circuit model can adjust using a very few parameters the laterals modes that appear in rectangular shaped FBAR or SMR resonators. Note that, as the LTLs are placed in series in the acoustic part of the Mason model, an equivalent circuit of the LTLs could be placed in series into the acoustic branch of a BVD model just replacing the value of the coupling transformer.

The proposed model of LTLs can be extended to simulate the effect of a border ring just cascading sections of evanescent transmission lines to the LTL presented in this work.

This work was supported in part by the Spanish Interministerial Commission on Science and Technology and the Europ. Regional Develop. Fund (FEDER) under Projects MAT2011-29269-C03-02 and TEC2012-37582-C04-02

\section{References}

[1] T. Pensala and M. Ylilammi, "Spurious Resonance Suppression in Gigahertz-Range ZnO Thin-Film Bulk Acoustic Wave Resonators by the Boundary Frame Method: Modeling and Experiment", IEEE Transactions on Ultrasonics, Ferroelectrics and Frequency Control, vol. 56, no. 8, pp. 1731-1744, Aug. 2009

[2] T. Jamneala, P. Bradley, U. B. Koelle, A. Chien, "Modified Mason Model for Bulk Acoustic Wave Resonators", IEEE Trans. on Ultras., Ferroelec. and Freq. Control, vol. 55, no. 9, pp. 2025-2029, Sept. 2008

[3] F. Abdukadirovna. "Dispertion Relations for Elastic Waves in Plates and Rods", MS Thesis, New Jersey, Jan 2011

[4] D. Royer, E. Dieulesaint, "Elastic Waves in Solids I, Free and Guided Propagation", Springer, 2000

[5] K.Y. Hashimoto, "RF Bulk Acoustic Wave Filters For Communications" Artech House 2009 